\title{
18
}

\section{IN AND MOBILITY}

\author{
Terje Jensen \\ Telenor Research and Development \\ Instituttveien 23, Postboks 83,N 2007 Kjeller, Norway \\ Tel: +47638091 00, Fax: +4763810076 \\ E-mail: terje.jensen@tf.telenor.no
}

\begin{abstract}
Intelligent Network (IN) is a platform for supporting telecommunication services. Mobile services are expected to be a significant fraction of the service demand, counted in number of users and the number of calls. Therefore, the support of such services by IN are considered with interest by several organisations. This is also valid for the use of IN to allow for service differentiation by the mobile network operators.

In this paper, classes of mobile services are defined and the corresponding requirements from each of these stated on the network capabilities are given. It is then indicated how IN has the potential to handle these services.
\end{abstract}

\section{INTRODUCTION}

Mobile services are among the most intensive increasing markets in telecommunications. Several forecasts have predicted that these services will still be handling a larger part of the users as well as the number of calls in the future. Still, however, much have been invested in the telecommunications infrastructure. Therefore, as faced by the operators, the question of how to utilise the equipment in a better way becomes more pronounced. This is understood in the sense that several services and load can be handled by the installed network. In some ways the mobile services could be described as providing added value to the basic services. On the other hand, several basic aspects could be requested for such services leading to other sets of requirements for the telecommunication network capabilities.

Intelligent Network (IN) is a concept which seems to be introduced in most networks, mainly because of the possibilities for rapid service deployment and flexible allocation of functionality. Two other factors associated with IN are the separation of the control/service domain from the switching/transmission domain and the use of building blocks to compose 
services. Both of these aspects will support the construction of more services and service features. Basically, we may say that IN could be a platform for offering services when the focus has been placed on the operators/providers role.

A number of other roles can be identified as well. The main roles are the service subscriber, service user, access provider, network operator and service provider. The service subscriber makes a contract with a service provider and is economical responsible for one or more users covered by the subscription. The user is authorised by the subscriber to utilise certain services within specific limits. An access provider handles the equipment which enables the users to access their services. A network operator is, as a minimum, responsible for the exchanges and transmission equipment. More equipment could also be provided, like control nodes and data bases. A service provider is responsible for the subscriber handling and data base containing subscriptions and users. Several of these roles could be carried out by the same person/company. For instance, the service provider and the network operator could be the same company (vertical integration). A number of other roles can be identified as well, like the regulator, service designer and equipment manufacturer.

As seen from IN, mobility could be supported by defining appropriate sets of services. As will be described later, a number of other elements could also be required for some implementations of mobile services. Often, the phrase "any one, any where, any time and any form" has been seen when describing the future of such services. This do allow for increased flexibility for the users. On the other hand, as seen from the operators/providers, the combination of IN and mobility is also very interesting. A number of further applications could be introduced and exposed to the market.

In this paper the relationships between IN and mobile services will be examined. That is, the focus is placed on the fixed network side. On the other hand, mobile services are often associated with wireless access. Aspects related to that portion of the service provision are not treated here.

The objectives of this paper are to clarify a number of terms related to mobility and describe some possible implementations for supporting these services. As the title reveals, a specific weight is placed on the IN based implementation. Naturally, other ways of providing the services could also be thought of.

In Section 2 the mobility classes are described. In the following two sections the two main classes of mobility, terminal mobility and personal mobility, are treated in more detail. A few aspects of the existence of the various mobile services and the use of IN are looked at in Section 5 , before some conclusions are given in the last section.

\section{CLASSES OF MOBILITY}

Mobile services can be classified into several main groups. Depending on the criteria used, these could be further divided into subgroups. This is only illustrated for terminal mobility. 


\subsection{Terminal mobility}

Although terminal mobility often is interpreted as involving a radio connection, a broader categorisation is done in the next points, e.g., ref. [E.50301]:

- Fixed: That is, no mobility at all. In the widest sense, it is not possible to disconnect the terminal from the access. No requirements are imposed on the network capabilities after the initialisation of the configuration.

- Portable: The terminal can change access points and will assume the identity and the profile of the access point. No additional requirements are imposed on the network capabilities as the access points carry the identifications as seen from the network side.

- Movable: The terminal keeps its identify after it has been moved between access points. Access to the services is not possible while moving between access points. This implies that the network must have capabilities for mapping between the terminal identity and the access identity. In addition, more information associated with the terminal identity could be stored in the network.

- Mobile: The terminal keeps its identity and the possibility to access the services also while moving. In general, this requires that the network has capabilities for knowing the terminal whereabouts.

We may define the level of terminal mobility to increase from the fixed (no mobility) to mobile. Therefore, the mobile terminal includes the others as subsets considering the requirements stated on the network capabilities. Mobile terminals also imply the use of wireless access. That is, when the services are available while moving, a wireline can not connect the terminal to the network.

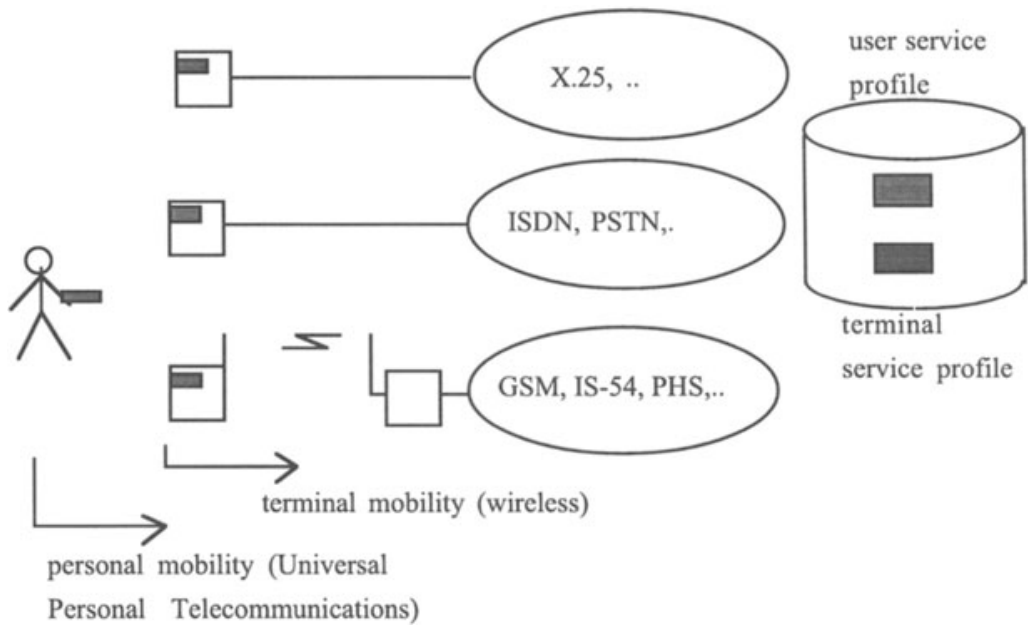

Figure 1 Illustrating different levels of mobility. 


\subsection{Personal mobility}

In case of personal mobility, the user (person) has an identity associated. This allows, for instance, a call to be directed to a user and not to the terminal where the user is supposed to stay. Moreover, the user can utilise a number of terminals in order to access the telecommunication services. Terminals of all the mobility subclasses could support personal mobility.

In order for the network to provide this mobility, some capabilities for mapping between the personal identity to the terminal/access identity must be present. From this it also follows that information of the users' whereabouts must be available in the network. Personal mobility may also be defined with several levels, each adding requirements for functions implemented in the network.

\subsection{Service mobility}

The feature named service mobility is often seen to include the personal mobility for more than one network. In addition, the user has access to his/her own defined set of services from different access points (and networks). Depending on the information given in the service subscription, limited versions of the services could be available in some networks. Naturally, this could further be restricted by the capabilities of the network and the current terminal.

The requirements on the network capabilities are similar to those resulting from personal mobility when considering the mapping possibilities. In addition, the user defined services must be available through the networks which are covered by this mobility feature.

\subsection{Relationships between mobility classes}

The different mobility classes could be thought of as sets. Then, often, service mobility is included in the set called personal mobility. That is, service mobility implies that personal mobility is present. On the other hand, terminal mobility could be supported without implementing personal mobility and vice versa. This means that terminal mobility and personal mobility is partly overlapping, however, having non-overlapping parts as well.

A schematic illustration of the mobility classes is given in Figure 1. A few networks are depicted with belonging terminals and access points. As stated earlier, terminal mobility often is associated with wireless access. As personal mobility is associated with the user, several terminals could be utilised by the user in order to access the services. These terminals have wireless or wireline connections to the corresponding networks. In this sense, personal mobility could be thought of as a higher level of mobility compared to terminal mobility. A database is included to show that capabilities for storing and retrieving service profiles can be present. This may allow, for instance, the user to access similar set of supplementary services from the different networks. For example, when abbreviated dialling is subscribed to, the same abbreviated number could be used both from an GSM (Global System for Mobile communication) terminal and from a terminal connected to PSTN (Public Switched Telephone 
Network). For terminal mobility, some information about the terminal itself could also be requested.

We may also say that terminal mobility is provided in close relationship with the systems, that is, it is a major characteristics of the system. On the other hand, personal mobility can be looked upon as a service provided by one or more systems.

One of the challenges by personal mobility is to support a consistent user interface over the different networks. Considering the example of abbreviated dialling, the sequence of dialled digits should be the same in the different networks. In most cases, this means that the different networks must have access to the data about the user. Personal mobility also introduces a number of other questions, like how to treat the fact that more than one user could be registered to the terminal at the same time. In particular, the problems raised by feature interaction must be looked into. Flexible and user-friendly solutions are requested for several issues.

\section{TERMINAL MOBILITY}

Although several terminal mobility subclasses were described in Section 2.1, only the one implying wireless access will be treated here. This is also regarded as the most interesting one in this context. Terminal mobility in this sense can also be called continuos mobility as the services may be accessed while moving.

\subsection{Applications of terminal mobility}

Terminal mobility could be applied in a number of environments each described by a set of characteristics. Typical applications can be domestic (private houses, flats, etc.), business (offices, storage rooms, production halls, etc.), vehicular (busses, air planes, trains, etc.) and public coverage. The listed environments are not exhaustive and not clearly separated. A set of other criteria could also be used to describe the areas where wireless systems can be used. Immediately, we recognize that these areas may have different users densities and different radio signal propagation characteristics. This invites for the introduction of different coverage areas for base stations. For example, a indoor base station may cover an area of $10 \mathrm{~m}$ radius while a base station in a satellite may cover an area of some hundreds of $\mathrm{km}$ in radius. In between these ranges there may be several other sizes depending on the traffic demand and the geography.

Recognizing the various environments also invites for the presence of a number of types of operators. Typically, a domestic area would be covered by a (set of) base station(s) provided by the persons living in that area, like a family could own the base station covering the house they are living in. In a similar way, a company could provide the set of base stations needed to allow for wireless access for the employee of that company. A third type of operators could be those providing public services. This service provision, however, could be for a restricted geographical area as well as limited in other ways. With all these potential operators, there must be corresponding lists of authorisation, that is, which users that can be allowed to use 
the operators' base stations. For example, the neighbour may not be allowed to utilise the base station owned by a family.

A number of classes of mobile terminals may also be present. These could be classified according to the usage of services and access to base stations (authorisation list). Service usage is, naturally, strongly correlated to the capabilities of the terminal, like what kind of audio/video functions that are implemented, the available battery capacity, and so forth.

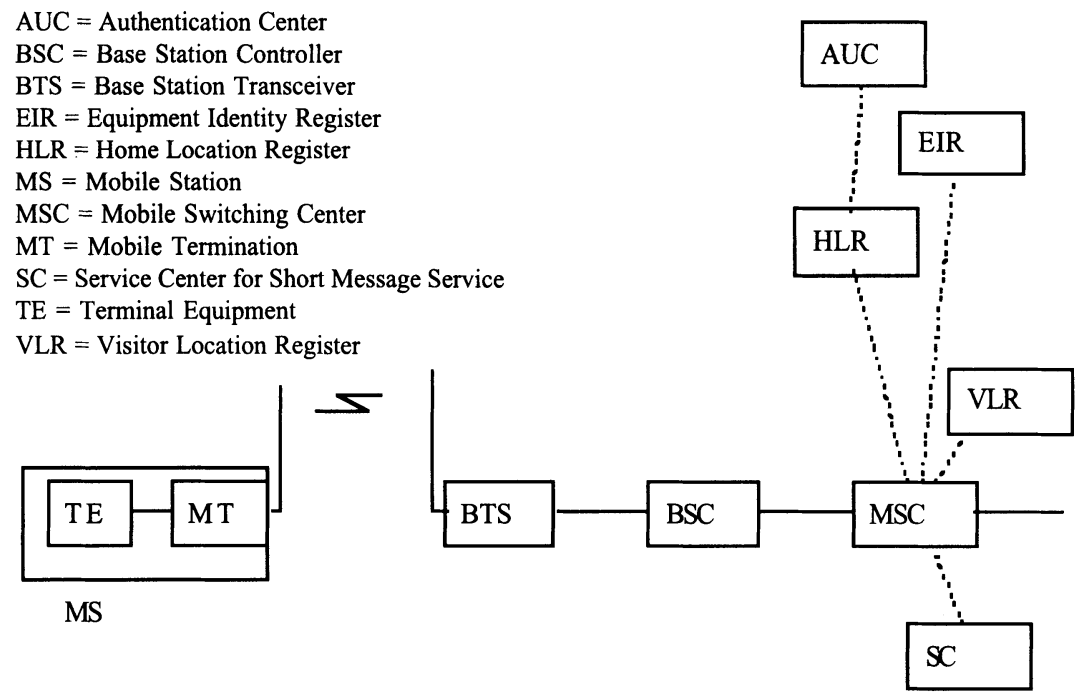

Figure 2 Example of entities present in a mobile system.

As the mobile terminal moves around between the different areas, often, the continuous use of telecommunications services is requested. This means that the functions supporting mobility in the different environments must interwork. As there may be several types of operators present, the definition of standard interfaces and procedures seems to be necessary. These are topics for the standardisation activities on future mobile systems.

The different wireless access systems present today support various sets of services. Therefore, it is simpler to compare the information bit rates the systems can provide. The more common digital mobile communications systems (e.g., GSM) can provide information bit rates of $9.6 \mathrm{kbits} / \mathrm{s}$ (data) or $13 \mathrm{kbits} / \mathrm{s}$ (voice). In some digital cordless systems (e.g., DECT, Digital European Cordless Telecommunication), several time slots could be utilised in order to arrive at information rates around a few hundreds of kbits/s. A single time slot in DECT, however, can support $32 \mathrm{kbits} / \mathrm{s}$. In future systems, like FPLMTS (Future Public Land Mobile Telecommunications Systems), around $2 \mathrm{Mbits} / \mathrm{s}$ for information rates are examined, e.g., see [R.8.1.95]. Even higher bit rates are also studied for some applications, like MBS (Mobile Broadband Service), e.g., see [Fern95]. This means that most of the services present 


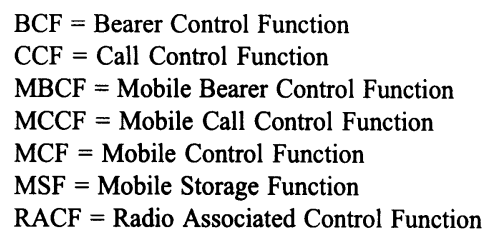

$$
\begin{aligned}
& \text { RBCF }=\text { Radio Bearer Control Function } \\
& \text { SCAF }=\text { Service Control Agent Function } \\
& \text { SCF }(M)=\text { Service Control Function (Mobile) } \\
& \text { SDF }(M)=\text { Service Data Function (Mobile) } \\
& \text { SRF }=\text { Special Resource Function } \\
& \text { SSF = Service Switching Function }
\end{aligned}
$$

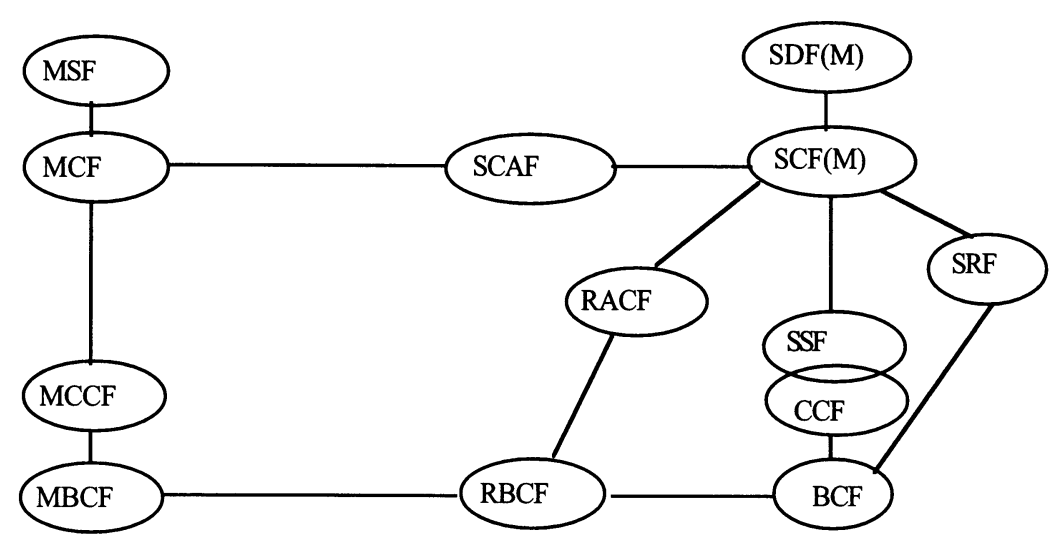

Figure 3 Functional model under examination for FPLMTS.

by wireline access also can be available by wireless access. In addition, mobile specific services, like location and navigation, could be supported. However, several topics must be further examined and a number of questions answered before such situations are reached. Naturally, all the services may not be available in all the environments outlined above. The highest bit rates may be provided in more local areas, while public access with traditional coverage areas may offer services supporting around 100 to $200 \mathrm{kbits} / \mathrm{s}$.

\subsection{Entities in mobile systems}

Several of the mobile systems have defined similar entities in order to support terminal mobility, as illustrated in Figure 2. Beginning from the user side, a terminal and mobile termination may compose a mobile station. This can be connected to a base station by a radio interface. A base station subsystem can be composed of a base station transceiver and a base station controller. Several base station transceivers may be connected to one base station controller. A number of base stations are connected to a switching node (exchange). In addition to these basic entities, others including the necessary control and data base functions are usually present. Still more entities have been described as well, like the short message service center in GSM.

The functional model presented for systems supporting terminal mobility depends on the capabilities of the system. In Figure 3 one model proposed for FPLMTS is depicted. 
Basically, we may recognize the IN functional model with SDF, SCF, SSF, CCF and SRF. In addition, the call and bearer (connection) may be separated. The bearer control functions are found in the lowest part in the illustration. Functional entities have been added because of the presence of a wireless access interface, like RBCF and RACF. There must also be functions capable of dealing with non-call related activities, like SCAF. These are typically used for mobility management. The different functional entities can be located in the terminal, access network and core network. However, a flexible allocation could be expected. Because of the capabilities of the mobile terminal, several of the functions in the network have a corresponding entity in the terminal. The control and data functions can be enhanced to support the mobile specific requirements. In the SSF we may find both a Basic Call State Machine (BCSM) and a Handover State Machine. The latter models the bearers in a way similar to the way the BCSM models the call and is, again, an effect of the mobility of the terminal.

\subsection{Terminal mobility procedures}

Because of the fact that mobile terminals can move around, a number of procedures must be enhanced or added compared to the non-mobile terminals. Some additional of such procedures are needed for:

- Call set-up: As the terminal may move, functions for locating the terminals before the call can be established are needed. This includes data bases and corresponding control functions.

- Handover: When the mobile, having an established call, finds that the quality of the connection towards the base station in use is below a specified threshold, a new base station may be taking over the call.

- Location update: A mobile without an established call may find that it has changed to a new geographical area (location area) and informs the network about this event.

- Paging: When a call is initiated towards a mobile terminal, the network must page for the terminal in order to locate it within the preregistered location area.

- Authentication: As the mobile terminal changes its connection point towards the network there is a need to make sure that the terminal and the network are the ones they pretend to be.

- Attach/Detach: In order to reduce the number of unsuccessful paging messages for terminals that are turned off, the terminal could send an indication of its change of status when turned on/off.

Each of these procedures implies additional requirements to the telecommunication network compared to when only wireline terminals are connected. Other mechanisms for interconnectivity could also be relevant as mobiles may access the network through different operators. In particular, arrangements for charging and accounting must be defined.

In future systems the service control and data are expected to become more distributed. Simultaneous procedures will also be required, like several services and mobility procedures 
could be active at the same time. In order to allow for improvement of the resource usage after handovers, bearer and call control should be more clearly separated. In addition, non-call related procedures must be allowed for. All these aspects have impact on the evolution of IN.

\section{PERSONAL MOBILITY}

Universal Personal Telecommunications (UPT) will be used for the discussion of personal (user) mobility. We may say that UPT arose from merging two telecommunications networks capabilities, that is, wireless access and intelligent network services. The first should allow for some mobility while the latter refers to UPT as a service based on a telecommunications platform. UPT can also be said to offer discrete mobility in the sense that specific actions are needed when changing connection points towards the network in order to still be accessible. For instance, the user may need to explicitly register on a terminal.

\subsection{Personal mobility features}

Some reasons for introducing UPT are to allow for increased flexibility, personification and availability (according to the user's requests). These may be achieved by utilising features like, e.g., ref. [T.F.850]:

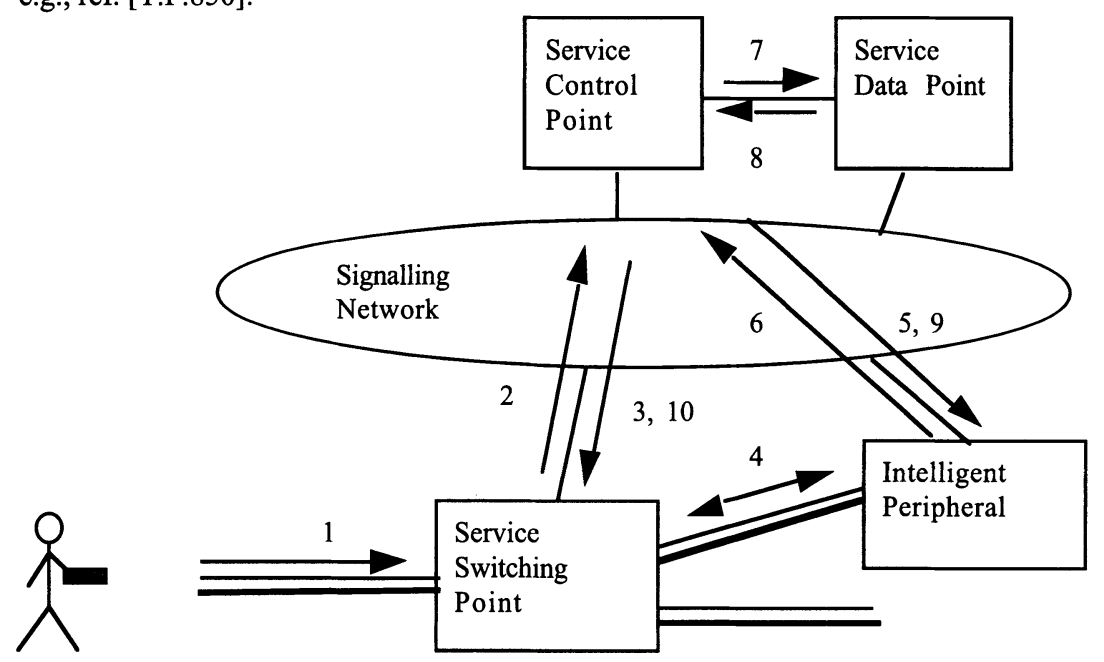

Figure 4. Sequence of interactions for registration to a terminal. 
- Personal numbering: A unique number identifies the UPT user and is used by the caller to reach that UPT user. That is, the number is associated with the user and is transparent for the networks supporting UPT.

- Service profile: The profile can include a user defined set of services. That is, a list of services and facilities subscribed to by the subscriber on behalf of the user.

- Access from multiple networks: The service is available from several networks and there are common service control procedures for all these networks. However, support of the services in the service profile may be restricted by the terminal and the network operator/service provider as well as the subscription.

- Terminal independence: The user may dynamically register to terminals. A user may also be registered to several terminals for the different services.

- Security and privacy: The first term may include the use of passwords and other mechanisms for preventing that non-authorised persons are accepted to be the user. Also specific charging and barring facilities may be relevant. To take care of the privacy includes minimising the risk for revealing personal data and incorrect charging. In addition, third parties (e.g., terminal owners) should not be too disturbed by the UPT users' activities.

- Access device: Such a device may be used for atomising the interaction between the user and the network. The device may take different forms and be incorporated into equipment for other purposes. Programmable DTMF (Dual Tone Multi Frequency) transmitters are one way of implementing such devices.

\subsection{Personal mobility procedures}

In order to allow the UPT user utilising the services, a number of procedures have been defined. These are grouped into three categories named handling of personal mobility, UPT call and UPT profile management. Several procedures can be identified for each of these groups, like registration for incoming calls and registration for outgoing calls in the group named handling of personal mobility. The third group is special in the way that the service profile can be interrogated and modified. That is, how the service is provided by the network can be changed by the user. All these procedures involve the user. For several of them, activities not related to a user-to-user connection are defined, like between the user and the SCP, alternatively with an IP included in some phases of the activity.

The procedures are composed of a number of steps, starting from the access and finishing when the session ends. The access can be done by the user dialling the UPT access number. Then, the identity of the user should be given, e.g., by keying it on the pad of the phone set. In addition, the user authentication code must be provided by the user. After the combination of identity and authentication code has been verified, the user can continue by selecting the action to perform, which may be from one of the three groups described above. However, others or packages of actions could also be selectable. Ending that action, the session could be finished or a request for performing another action could be chosen (named Follow-on option).

A number of telecommunication network elements could be involved during these procedures, see Figure 4. The SSP would recognize the UPT access number and send a 
message to the SCP (2 in Figure 4). A reply could tell the SSP to establish a connection to an IP for translating the dialled UPT identity and authentication code from Dual Tone MultiFrequency (DTMF) signals to digits. These digits can be sent from the IP to the SCP (6 in Figure 4) which composes a query to a SDP in order to verify the user provided information. The procedure can continue with the exchange of several messages and the involvement of more network elements.

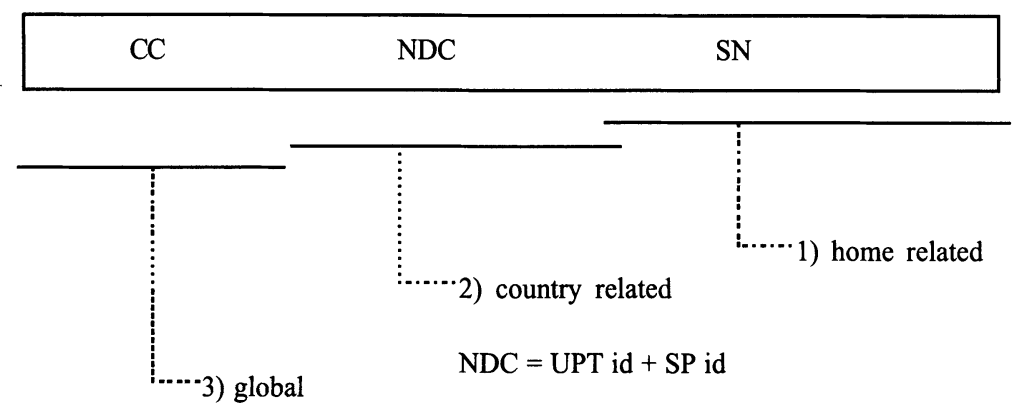

a) $\mathrm{CC}(\mathrm{UPT})+\mathrm{NDC}($ non-CC) $+\mathrm{SN}$

b) $\mathrm{CC}(\mathrm{UPT})+\mathrm{NDC}(\mathrm{CC})+\mathrm{SN}$

$\mathrm{CC}=$ Country Code

NDC $=$ National Destination Code

$\mathrm{SN}=$ Subscriber Number

SP id = Service Provider indicator

UPT id $=$ UPT indicator

Figure 5 Possible numbering schemes for UPT.

Summing the number of digits provided by the user during such a session, we may find that this could be in the range of 25 - 30 digits just for making an outgoing call (without previous registration). In order to alleviate this procedure for the users, access devices seem to be requested. In addition, we see that a lot more signalling and processing are needed to serve the user requests. Data base look-ups could also be carried out during some phases of the call handling.

\subsection{Network functions}

The data related to a user could be stored in a user profile. In addition to keeping the identity and possibly the authentication code, other information for personalising the services could be given. This could, for instance, include routing tables, charging arrangements, customized voice 
announcements, and so forth. In particular, routing tables could allow for defining default accesses where the user usually stays at certain periods of the days. References to voice mail or other answering services could also be included.

Different numbering schemes for UPT are found in [T.E.168], see Figure 5. A number can be composed of a country code (CC), a national destination code (NDC) and a subscriber number (SN). Three schemes are defined based on these fields:

1) Home related: That is, the difference between the number of an UPT user and other users can only be found by looking at the subscriber number field. This implies that only the home network of the UPT user can recognize that it is a call to an UPT user.

2) Country related: That is, the NDC field can be composed of an UPT indicator and an service provider indicator. The first will only tell that this is an UPT user, while the latter identifies the provider of the UPT service.

3) Global: That is, an indication of the UPT number is found in the country code field. This number can then be recognized in all the networks supporting the UPT service.

As an alternative to these schemes, the use of a special prefix has been described. By dialling this prefix, it is directly indicated that the call is for an UPT user. Which scheme that is chosen, will influence the possible control architectures as returned to later.

The requirements stated by the UPT service on the network capabilities can be listed as:

- Recognizing the selected numbering plan: That is, the UPT numbers and the UPT procedures must be recognized.

- Dynamic registration of users to access points/terminals: This means that dynamic linking between the user and access points/terminals must be possible, and information about how to reach the user must be available. It also includes the use of authentication in order to ensure that the user is still present in case a call is presented at the given terminal.

- Access from multiple networks: This may imply the exchange of information between the visited network and the home network of an UPT user. Proper procedures for this have to be established. In addition, consistent user interface should be achieved.

- Storage of user specific service data: In particular, service profile should be allowed for in order to personalise the service.

- Flexible charging/billing arrangements: Special arrangements could be requested, like credit limits for a period, split charging, etc. Procedures for accounting between operators could also be needed.

These are basic requirements on the networks supporting the UPT service. Others could also be given that may increase the usability of the service, like the support of access devices. We may find that the use of IN could fulfill these requirements. However, a number of selections and arrangements must be established, in particular, when several networks and operators are involved. 


\section{SUPPORT OF MOBILITY SERVICES}

Several services could be integrated in a telecommunications network in a number of ways. The most basic integration level may be common use of equipment, like the services are handled by the same exchange. Then, additional integration schemes like common signalling and functions can be defined. Although achieving a high level of integration may seem to be requested from an economical point of view, it could imply that several trade-offs must be done. This may again mean that the resulting solution might not be the better one for any of the services/applications.

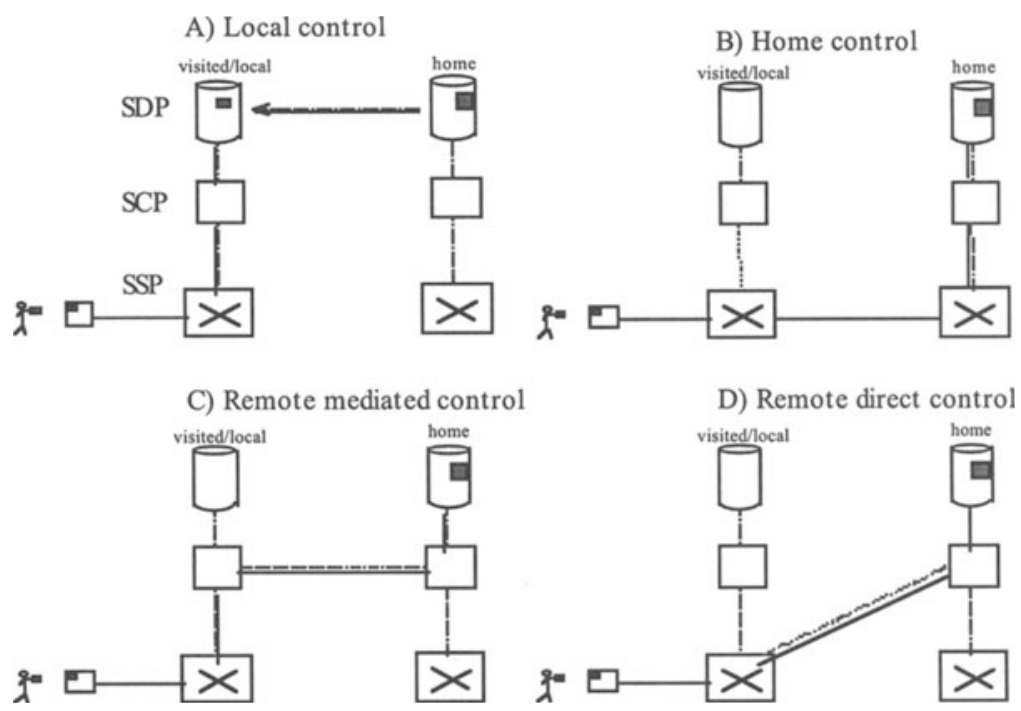

Figure 6 Some possible architectures for providing the service through a visited operator's network.

Another question is how the services are supported by a sequence of network operators, that is, how to handle the fact that users may want to access the services through the network handled by another operator. Some cases for enabling the support of services in several network have been defined. These could then be compared according to a set of evaluation criteria.

The use of standardised service profiles and service logic could be regarded as an advantage, but may also restrict the possibilities for operators to introduce better service features in the competition with others. This may also complicate the deployment of services when different 
versions of the network capabilities are present. Standardised profiles may allow them to be transferred between the different operators/providers. On the other hand, such transfers do also mean that customer information is revealed to the other operator/provider. The use of standardised interfaces is usually preferred. In case a solution requires the presence of an interface not treated in any standards, special arrangements must be agreed upon between the two parties. In particular, this must be treated in more detail when one operator is able to control the equipment provided by another operator.

The user may notice the interface, the set of services available and the quality of the services. The last one includes the delays for the procedures involved. Normally, the involvement of several elements leads to an increased delay. Longer signalling relations and connections may also imply a higher cost as seen from the operator side.

Different cases can be illustrated by looking at the configuration arising when a user, having a subscription in the home network, accesses the services through a visited network, see Figure 6. Some architectures for supporting the services over more network operators can then be classified like:

A) Local control: Where the service profile is transferred to the visited network when the user is registered in the visited network. That is, a relation between the two data bases is assumed.

B) Home control: Where the service is controlled in the home network. This implies that a connection to the home network must be established.

C) Remote mediated control: Where the service execution is controlled from the home network. The service control messages are mediated by the local service control.

D) Remote direct control: Where the service execution is controlled from the home network by direct accessing the switching functions in the visited network.

The different architectures can be compared according to the criteria outlined above. We find that local control could require that service profiles are standardised and that customer information will be revealed. Thus, allowing for special services and new versions could be more difficult. Home control may result in long connections although it may be better according to other criteria concerning independence and security. Remote direct control allows another operator to set parameters in the SSP, which implies that the corresponding call handling at that level must be defined.

\section{CONCLUSIONS}

Mobile services are among the telecommunication services with highest annual growth. As described above, these may be classified as terminal and personal/service mobility. These classes are not disjoint. However, as they provide mobility, we may face situations where they are competing in order to serve the demand. Some characteristics are relevant for each of these groups: As wireless access utilises radio propagation, the capacity is normally restricted (counted in kbits/s and number of users active simultaneously). Personal mobility is supposed to cover several networks including wireless. Personal mobility is associated with discrete 
mobility, therefore certain activities are requested from the user in order to achieve this mobility. For this, the use of access devices might be crucial, otherwise the procedures could be rather heavy. To a certain extent, this could be alleviated by the use of wireless access services for informing the network about the user's location. After knowing this, the network could have corresponding attributes given in service profiles and algorithms to select the most appropriate way of utilising the telecommunication network when a service is activated.

IN is a concept under standardisation which enables interoperability and provides an interface towards the user. In addition, management systems have been defined in order to support the services provided by the IN platform. UPT, which has been an implementation of personal/service mobility, can be regarded as a service executed on the IN platform. Several mobile networks have introduced a concept very similar to IN. Access to IN-based services through wireless is also regarded as a necessity in order to allow for further diversification for the operators.

As, by nature, mobile services allow the users to change locations, appropriate capabilities in the network must be defined to support these activities. This include the dynamic updating of location and routing. Furthermore, authentication procedures are necessary because the user is not identified by the access point that is used. Individualised service profiles and charging/billing arrangements are also requested. This implies that much data could be needed for providing the services, also data associated with each user.

Because an advanced level of service control is anticipated, the control activity could be allocated to separate nodes. According to the conceptual model, Intelligent Networks meet these requirements. However, some enhancements are foreseen to support the mobile services. How to include these features in the IN concept, is examined in the standardisation organisations.

It is expected that further services will be based on IN and that more systems will adapt to the IN concept. Naturally, other concepts may replace IN after a while, but the mobile services have to be taken into account for all the service control platforms.

\section{REFERENCES}

[E.50301] ETSI Technical Report Draft-ETR/SMG 50301: Framework of network architecture, interworking and integration for the Universal Mobile Telecommunications System (UMTS). Version 0.2.3. June, 1992.

[Fern95] Leandro Fernandes: Developing a System Concept and Technologies for Mobile Broadband Communications. IEEE Personal Communications. Feb. 1995, p. 54-59.

[R.8.1.95] ITU-R: FPLMTS/IMT-2000. Report of the Ninth Meeting of ITU-R Task Group 8/1. Tokyo, Japan. Sept. 1995.

[T.E.168] ITU-T rec. E.168: Application of E.164 numbering plan for UPT. Geneva, 1993.

[T.F.850] ITU-T rec. F.850: Principles of Universal Personal Telecommunication (UPT). Geneva, 1993. 


\section{BIOGRAPHY}

Terje Jensen, Ph.D., is currently working as research scientist in the strategic network development group at Telenor Research and Development. The work interests include performance models and analyses of telecommunication networks and services. In particular network dimensioning issues, with main emphasis on Intelligent Networks, have been considered so far. Prior to receiving his Ph.D. degree he was also involved in administration and operational support for introduction of digital exchanges while working as senior engineer in Northern region of Norway. He has taken part in several international projects related to network dimension issues. 